\title{
Design of a Lane-Tracking Driver Steering Assist System and Its Interaction with a Two-Point Visual Driver Model
}

\author{
Spyridon Zafeiropoulos
}

\begin{abstract}
In this paper we investigate the design of a lanetracking driver-assist system and, in particular, its interaction with the well-known two-point visual perception driver model. We present two different driver-vehicle interconnections and two different control designs that assist the driver in the lanetracking task; the first design is based on linear multivariable output regulator theory, and the second design is based on model predictive control (MPC) theory. In the first interconnection the controller is "blind" to the driver's actions, and an appropriate blending of the controller and driver actions is used in order to steer the vehicle. In the second interconnection the control design takes into consideration the driver model, and the control steering command is shared between the lane-tracking steering assist system and the driver. The performance of both controllers and interconnections is compared and evaluated using three different drivers and also against a unassisted driver-only scenario.
\end{abstract}

\section{INTRODUCTION}

The idea of autonomous steering has long been a popular research area in the automotive industry due to its applicability to passenger cars for active safety and comfort (e.g., autonomous lane-following systems). One approach for automatic roadway tracking is presented in [1], where the authors introduced several design methodologies for both lane estimation and lane tracking. In [2] and [3] different control schemes for the design of autonomous lanefollowing/tracking control systems are given, and a lookahead distance is used in order to calculate the offset of the vehicle center of mass from the reference trajectory. An alternative approach promotes the use of lateral preview control [4], [5]. Even though these techniques provide excellent performance, their optimization-based methodology makes them often difficult to implement on-line.

Numerous vehicle steering systems designs exist in the literature, but works investigating the interaction between the driver and the steering system are rather scant. In [6] the authors present a model that mimics driver behavior during a path following task. In [7] the author introduced the idea of the two-level driver model, where the steering task is divided in two levels, an anticipatory open-loop control mode and a closed-loop compensatory mode that handles deviations from the reference. The idea that human drivers use a two-tier perception system while driving has also been confirmed by several studies, especially in the pioneering

S. Zafeiropoulos is a graduate student at the School of Aerospace Engineering, Georgia Institute of Technology, Atlanta, GA 30332-0150, USA, Email: spyros.zaf@gatech.edu

P. Tsiotras is Dean's Professor at the School of Aerospace Engineering, Georgia Institute of Technology, Atlanta, GA 30332-0150, USA, Email: tsiotras@gatech.edu

\author{
Panagiotis Tsiotras
}

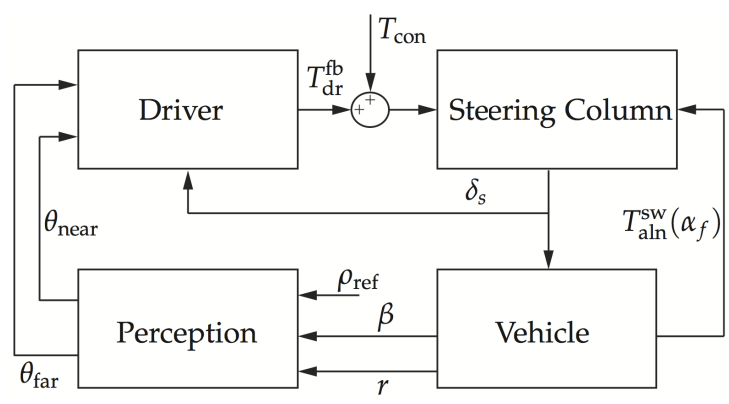

Fig. 1. The interconnection between the different subsystems: (a) vehicle, (b) motion and vision kinematics, (c) driver, and (d) steering column.

work of M. Land and his colleagues [8], [9]. More recently, Salvucci [10] developed a two-point visual control model, in order to steer the vehicle. The authors in [11] developed a complete model for lateral steering control that integrates many of the previously existing driver models. Additionally, the authors in [12] presented a control design based on the driver model by [6]. Similarly to the current paper, their objective was assisted lane-tracking. However, they achieved this objective with the use of active-steering and yaw moment control, instead of a torque-based steering assist system as is proposed in this paper. Furthermore, the authors in [12] used a simple driver model that does not take into account the two-point perceptual driver input. Finally, the authors in [13] use a two-point visual driver model along with a preview controller to perform assisted lateral steering. A robustness analysis in terms of driver parameter uncertainty is also provided.

In this paper we investigate the active connection between a lane-tracking control system and an existing driver model and evaluate their interaction. We are particularly interested in the way the driver's steering effort changes in the presence of a lane-tracking steering-assist system compared to the unassisted case after incorporating a perceptual/physiological driver model as part of the control design. We evaluate two different control designs, one based on multivariable output regulator theory (ORT), and the other based on model predictive control (MPC). Either of these two lane-tracking assist systems operates in parallel with the driver. The purpose of each controller is to observe driver tracking errors and, if necessary, intervene to correct them. Our numerical simulations using a high-fidelity vehicle simulator [14] show the importance of the seamless interaction between the lanetracking assist system and the driver when compared, say, with the case of a lane-tracking assist system that is oblivious to the driver. 


\section{Vehicle And RoAd Modeling}

The driver/vehicle system consists of four different subsystems (see Figure 1), namely: (a) the vehicle, (b) the perception and vision kinematics, (c) the driver, and (d) the steering column. In Figure $1 T_{\mathrm{dr}}$ is the driver torque command and $T_{\text {con }}$ is the torque command from the steering assist system. Manual vehicle operation corresponds to $T_{\text {con }}=0$. The details for the four subsystems shown in Figure 1 are briefly discussed below.

\section{A. Vehicle Dynamics}

The vehicle model is based on the linearized singletrack model, or bicycle model [15] (see also Figure 2). The

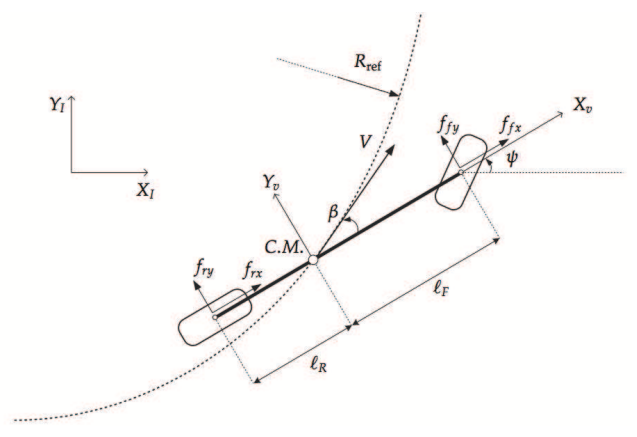

Fig. 2. Schematic of the bicycle vehicle model.

linearized vehicle dynamics are given as follows

$$
\begin{aligned}
& \dot{\beta}=-\frac{C_{r}+C_{f}}{m V_{x}} \beta+\left(-1+\frac{\ell_{R} C_{r}-\ell_{F} C_{f}}{m V_{x}^{2}}\right) r+\frac{C_{f}}{m V_{x}} \delta, \\
& \dot{r}=\frac{\ell_{R} C_{r}-\ell_{F} C_{f}}{I_{z}} \beta+\left(-\frac{\ell_{R}^{2} C_{r}+\ell_{F}^{2} C_{f}}{I_{z} V_{x}}\right) r+\frac{\ell_{F} C_{f}}{I_{z}} \delta, \\
& \dot{\psi}=r,
\end{aligned}
$$

where $\beta[\mathrm{rad}]$ is the vehicle sideslip angle, $r[\mathrm{rad} / \mathrm{sec}]$ is the vehicle yaw rate, $\psi[\mathrm{rad}]$ is the vehicle yaw angle, $V_{x}[\mathrm{~m} / \mathrm{sec}]$ is the longitudinal component of the velocity of the vehicle center of mass expressed in the vehicle frame, $m[\mathrm{~kg}]$ is the vehicle mass, and $I_{\mathrm{z}}\left[\mathrm{kg} \mathrm{m}^{2}\right]$ is the vehicle moment of inertia with respect to the vehicle mass center. In addition, $\delta[\mathrm{rad}]$ is the front wheel steering angle, $\ell_{F}[\mathrm{~m}]$ and $\ell_{R}[\mathrm{~m}]$ are the distances of the vehicle mass center from the front and rear axles, respectively, and $C_{f}>0[\mathrm{~N} / \mathrm{rad}]$ and $C_{r}>0$ $[\mathrm{N} / \mathrm{rad}]$ are the cornering stiffnesses of the front and rear tires, respectively, which are assumed to be constant. The vehicle dynamics interact with the steering column dynamics via the alignment torque $T_{\text {aln }}[\mathrm{Nm}]$, which is given by

$$
T_{\mathrm{aln}}=K_{\mathrm{aln}} \alpha_{f}=K_{\mathrm{aln}}\left(\beta+\frac{\ell_{F} r}{V_{x}}-\delta\right),
$$

where $K_{\text {aln }}=-K_{p} C_{f} \eta_{t}$, and where $K_{p}>0$ is the manual steering column coefficient and $\eta_{t}[\mathrm{~m}]$ is the tire length contact. The expression (1) has been shown to be valid for normal operating ranges and conditions, [16].

\section{B. Steering Column Dynamics}

The steering column system plays a significant role for capturing the interaction between the vehicle and the driver, and hence its modeling is imperative in a driver-assist system design. The steering column dynamics can be described by

$$
\begin{aligned}
J_{s} \dot{\omega}_{s} & =-b_{s} \omega_{s}-T_{\mathrm{aln}}^{\mathrm{sw}}+T_{\mathrm{dr}}+T_{\mathrm{con}}, \\
\dot{\delta}_{s} & =\omega_{s},
\end{aligned}
$$

where $\omega_{s}[\mathrm{rad} / \mathrm{s}]$ is the steering angle rate, $\delta_{s}=\delta g_{s}[\mathrm{rad}]$ where $g_{s}$ is the gear ratio, $b_{s}[\mathrm{Nms} / \mathrm{rad}]$ is the friction coefficient of the steering column, $J_{s}\left[\mathrm{kgm}^{2}\right]$ is the steering column moment of inertia, $T_{\mathrm{dr}}[\mathrm{Nm}]$ is the torque provided by the driver, $T_{\text {con }}[\mathrm{Nm}]$ is the corrective torque provided by the control system, and $T_{\text {aln }}^{\mathrm{sw}}=T_{\mathrm{aln}} / g_{s}$.

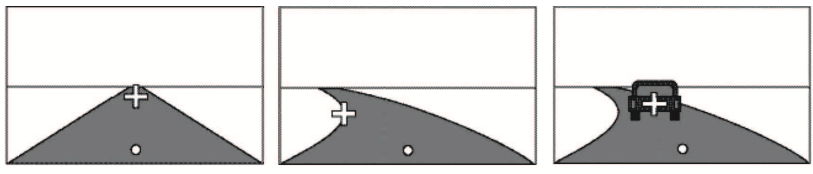

Fig. 3. Several studies of human steering strategies have revealed that drivers use a combination of a near feature point (white dot) and a far away feature point (white cross) to establish lateral stability and lane tracking; from [10].

\section{Road Geometry and Road Kinematics}

The coupling between the vehicle model and the driver is achieved via the road model, which provides the road geometry and the kinematics of the reference trajectory induced by the motion of the vehicle. In the sequel it will be convenient to use the curvature of the road, $\rho_{\text {ref }}=1 / R_{\text {ref }}$, as the input generating the reference path [3], [7], [11], [15].

The vehicle motion is modeled assuming small deviations from a stationary circular (or straight) path. To this end, and following [15], let the road's state vector be given by $x_{r}=$ $(\Delta \psi, \Delta y)^{\top}$, where $\Delta \psi=\psi_{t}-\psi[\mathrm{rad}]$ is the angle between the tangent to the path at point $\mathrm{A}$ and the vehicle heading and $\Delta y$ is the distance of a "lookahead" point at a distance $\ell_{s}$ $[\mathrm{m}]$ in front of the vehicle center of mass from the reference road trajectory (see Figure 5). It can be easily shown that the rate of change of $\Delta y$ is approximately given by

$$
\Delta \dot{y}=-V_{x}(\beta-\Delta \psi)-\ell_{s} r+V_{x} \ell_{s} \rho_{\text {ref }},
$$

while $\Delta \psi$ obeys the differential equation

$$
\Delta \dot{\psi}=\dot{\psi}_{t}-\dot{\psi}=r_{t}-r,
$$

where $r_{t}=v_{t} / R_{\text {ref }}=v_{t} \rho_{\text {ref }}$ is the yaw rate along the path tangent. Subsequently, and since $v_{t} \approx V_{x}$, it follows that

$$
\Delta \dot{\psi}=V_{x} \rho_{\text {ref }}-r .
$$

Finally, the kinematic road model is written as

$$
\begin{aligned}
\dot{x}_{r} & =A_{r} x_{r}+B_{r 1} u_{r}+B_{r 2} \rho_{\text {ref }}, \\
y_{r} & =C_{r} x_{r},
\end{aligned}
$$

where $u_{r}=(\beta, r)^{\top}, y_{r}=(\Delta \psi, \Delta y)^{\top}$. Note that in this model, the road curvature $\rho_{\text {ref }}$ plays the role of an exogenous signal. 


\section{Two-Point Visual Tracking Driver Model}

The internal connections of the driver model can be seen in Figure 4. The inputs to the driver model are the two visual angles denoted by $\theta_{\text {far }}$ and $\theta_{\text {near }}$ provided by the motion and the vision kinematics, and the current steering wheel angle, while the output of the driver model is the driver torque, which is applied at the steering wheel. The angles $\theta_{\text {far }}$ and $\theta_{\text {near }}$ roughly correspond to the two visual points tracked by the driver's visual system (see Figure 3). Indeed, several psychological studies provide strong evidence that drivers focus their attention on two "anchor" points in their visual field of view. A faraway point, often at the periphery of the visual field, and typically on the inner side of the road while cornering, and a closer-by point at a small distance ahead, approximately at the center of the visual field [10]. On the other hand, the channel of the steering wheel angle takes into account the reaction from the road and is used to model the kinesthetic part of the driver model.

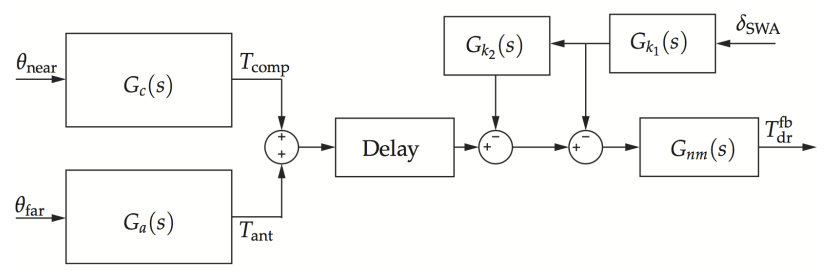

Fig. 4. The components of the driver model and their interconnection.

\section{A. Driver Model}

The driver model used in this study is based on the model developed in [11]. The model has five main components. Next, we give a brief overview of the model components.

The anticipatory control subsystem has transfer function

$$
G_{a}(s)=K_{\alpha},
$$

and accounts for tracking the reference trajectory at a distant future time. It is thus related to the far viewpoint, whose location is determined by the angle $\theta_{\text {far }}$ in Figure 5. The compensatory control subsystem is used for driving corrections in the near future, and it is thus related to the location of the near reference point via the angle $\theta_{\text {near }}$. It is assumed to have the transfer function

$$
G_{c}(s)=K_{\mathrm{c}} \frac{T_{L} s+1}{T_{I} s+1},
$$

where $T_{L}$ [sec] and $T_{I}$ [sec] are lead and lag time constants, respectively, with $T_{L}>T_{I}$. The delay with transfer function

$$
G_{L}(s)=\mathrm{e}^{-t_{p} s},
$$

accounts for the processing delay of sensory signals in the human brain. The neuromuscular subsystem models the driver's arms, and has the transfer function

$$
G_{n m}(s)=\frac{1}{T_{N} s+1} .
$$

The kinesthetic part that is comprised of two subsystems, $G_{k 1}(s)$ and $G_{k 2}(s)$, and accounts for the reaction provided to the driver by the steering system

$$
G_{k_{1}}(s)=K_{D} \frac{s}{s+1 / T_{1}}, \quad G_{k_{2}}(s)=K_{G} \frac{T_{k_{1}} s+1}{T_{k_{2}} s+1} .
$$

In the sequel, and for the sake of simplicity, only the subsystems (9)-(12) will be used for control design. Numerical simulations have shown that the kinesthetic part has a minor impact on the overall driver response and can be omitted without sacrificing the overall performance. Using a firstorder Padé expansion to approximate the delay in (11) with a rational transfer function, yields the following a state-space representation

$$
\begin{aligned}
\dot{x}_{d} & =A_{d} x_{d}+B_{d} u_{d}, \\
y_{d} & =C_{d} x_{d},
\end{aligned}
$$

where the driver state vector is $x_{d}=\left(x_{d 1}, x_{d 2}, x_{d 3}\right)^{\top}$, the input vector is $u_{d}=\left(\theta_{\text {near }}, \theta_{\text {far }}\right)^{\top}$, and the output is $y_{d}=$ $x_{d 3}=T_{\mathrm{dr}}^{\mathrm{fb}}$, where the matrices $A_{d}, B_{d}$ and $C_{d}$ can be easily calculated from (9)-(12).

\section{B. Visual Perception Geometry}

The last missing element to complete the driver model is to define the geometric characteristics of the vision/perception model, that is, how the driver perceives the road geometry and the vehicle's relative position through visual cues, namely the angles $\theta_{\text {far }}$ and $\theta_{\text {near }}$.

These two angles can be determined from the road geometry as shown in Figure 5. Specifically, $\theta_{\text {near }}$ is related to the near viewpoint of the driver $\ell_{s}$ and is given approximately by

$$
\theta_{\text {near }} \approx \frac{\Delta y}{\ell_{s}} .
$$

The angle $\theta_{\text {far }}$ is defined as the angle between the car heading and the gaze direction that is approximately tangent to the inner boundary of the road, and can be approximated by

$$
\theta_{\mathrm{far}} \approx \frac{L_{s}}{R_{r}}+\Delta \psi \approx L_{s} \rho_{\mathrm{ref}}+\Delta \psi,
$$

where for the last expression we have made the assumption that the vehicle distance from the inner road boundary (distance $d$ in Figure 5) is small compared to the road radius. Equations (16) and (17) clearly show the distinct role these two angles play in the feedback interconnection; $\theta_{\text {near }}$ can be viewed as a surrogate measurement of the lateral displacement $\Delta y$, whereas $\theta_{\text {far }}$ provides information about the road curvature [7], [11], [17].

\section{Lane-Tracking Steering Assist Control DESIGN}

In order to increase the lane-tracking performance of the vehicle/driver system and compensate for possible inadequate driver (re)action, we investigate the benefits of a driver steering-assist system, which injects a correction torque $T_{\text {con }}$ to the driver torque command $T_{\mathrm{dr}}$ as shown in Figure 1. We investigate two possible control designs. 


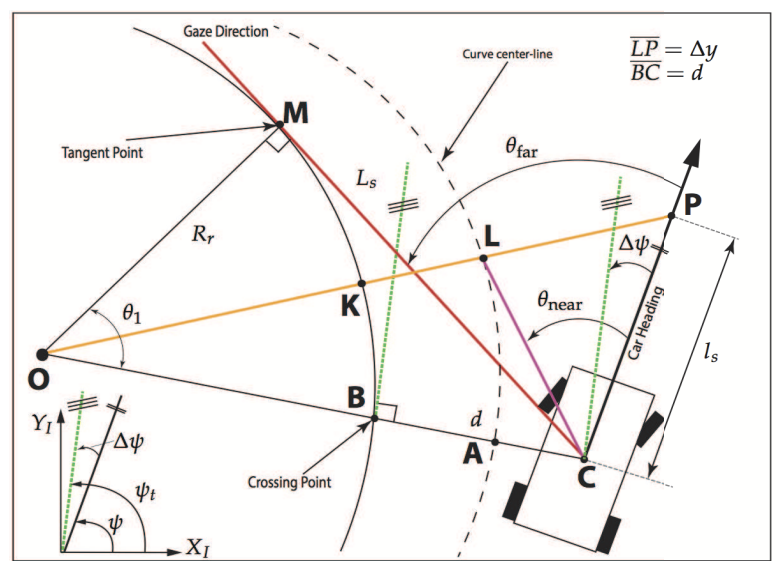

Fig. 5. The geometric relationship between the vehicle kinematics, the road reference trajectory and the driver's two-point visual model.

The "traditional" approach is to design a controller without taking into consideration the driver. In this approach, the total control torque command input to the steering column is given via a "blending" of the driver and control input commands as follows

$$
T_{\mathrm{tot}}=(1-\lambda) T_{\mathrm{dr}}+\lambda T_{\mathrm{con}},
$$

where $\lambda$ is the blending parameter taking the values $0 \leq \lambda \leq$ 1. For $\lambda=0$ only the driver command is active, whereas when $\lambda=1$ the vehicle operates completely autonomously. In the sequel, we will refer to this controller as the $\mathrm{BC}(\lambda)$ ("blending") controller. The second control design takes into account the driver model and thus operates on the combined vehicle/driver system. In this case the total input torque is

$$
T_{\mathrm{tot}}=T_{\mathrm{dr}}+T_{\mathrm{con}}
$$

In the sequel we will refer to this controller as the DiLC (Driver-in-the-Loop) controller. It should be noted, that the driver commanded torque is comprised of a feedforward term, $T_{\mathrm{dr}}^{\mathrm{ff}}$, in order to compensate for the alignment moment, and a feedback term, $T_{\mathrm{dr}}^{\mathrm{fb}}$ that is generated from the perception model, and therefore, $T_{\mathrm{dr}}=T_{\mathrm{dr}}^{\mathrm{ff}}+T_{\mathrm{dr}}^{\mathrm{fb}}$. It is assumed that torque blending is performed with the use of a steerby-wire system [18]. Consequently, at the implementation level, one has the freedom to steer the wheels by using any combination of the driver input torque and the steering-assist system torque, while feeding back to the driver a "sensing" torque that can be generated at will. Therefore, and for the sake of simplicity, in this work we will assume that the torque fed back to driver is equal to the alignment torque, that is, $T_{\mathrm{dr}}^{\mathrm{ff}}=T_{\mathrm{aln}}^{\mathrm{sw}}$.

For both cases, we have designed two different controllers; the first is based on the output regulator (ORT from now on) theory [19], and the second is a simple Model Predictive Control (MPC) [20] design with only input constraints.

\section{A. Output Regulator Design}

First, we re-write the state-space representation of the overall system as follows

$$
\begin{aligned}
\dot{x} & =A x+E w+B u, \\
\dot{w} & =0, \\
y & =C x=\Delta y,
\end{aligned}
$$

where $u=T_{\text {con }}$, and where the matrices $A, E, B$ and $C$, can be easily computed from the results of Sections II and III. In (20)-(22) the state is $x=\left(\omega_{s}, \delta_{s}, \beta, r, \psi, \Delta \psi, \Delta y\right)^{\top}$ for the case of the system without the driver, and $x=$ $\left(\omega_{s}, \delta_{s}, \beta, r, \psi, \Delta \psi, \Delta y, x_{d 1}, x_{d 2}, x_{d 3}\right)^{\top}$ for the case of the system with the driver included. In both cases, $w=\rho_{\text {ref }}$ is the (piecewise constant) exogenous signal, which is assumed to be measurable.

The objective is to design a controller to track the center lane at the look-ahead near point, that is, $\lim _{t \rightarrow \infty} \Delta y(t)=$ 0 . It is assumed that the control input is given as a linear combination of a feedback and a feed-forward term

$$
T_{\text {con }}=F x+G w .
$$

In order to compute the controller gain matrices $F$ and $G$ so as to achieve the asymptotic regulation of the output of (20)(22) to zero, we use output regulator theory [19]. Following the approach in [19] the matrix $F$ is chosen so that $A+B F$ is Hurwitz, and $G$ is chosen such that $G=\Gamma-F \Pi$ where $\Pi$ and $\Gamma$ solve the following matrix equations [19]

$$
\begin{aligned}
A \Pi+B \Gamma+E & =0, \\
C \Pi & =0 .
\end{aligned}
$$

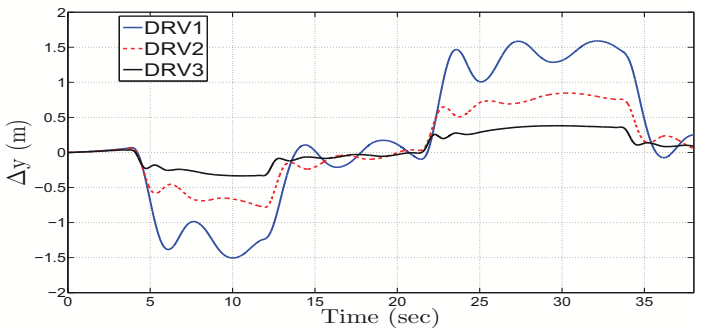

Fig. 6. Comparison of $\Delta y$ responses for the three drivers.

A standard LQR design has been used to find the stabilizing matrix $F$ in (23) with performance index

$$
J(u)=\int_{0}^{\infty}\left[x(t)^{T} Q x(t)+u(t)^{T} R u(t)\right] \mathrm{d} t,
$$

where $u=T_{\text {con }}$, and $Q$ and $R$ are the state and input weighting matrices, respectively. The matrix $R$ for all cases was chosen as $R=0.1$, while for $\mathrm{BC}(\lambda)$ the matrix $Q$ was $Q=25 \cdot \operatorname{diag}(1,0,10,0,0,10,10)$, and for the case of DiLC the matrix $Q$ was chosen as $Q=25$. $\operatorname{diag}(1,0,10,0,0,10,10,0,0,0)$. These choices reflect our objective to mainly regulate $\Delta y$ and $\Delta \psi$ (lane tracking), and $\beta$ (yaw stability). The steering angle rate $\dot{\delta}_{s}=\omega_{s}$ is also penalized to obtain moderate steering wheel command 
rates. The choice for $R$ was made so that adequate control authority was given to the steering-assist system in order to compensate for the driver's actions, while at the same time penalize the control action in such a way that the torque generated by the controller is of same order of magnitude as the driver torque.

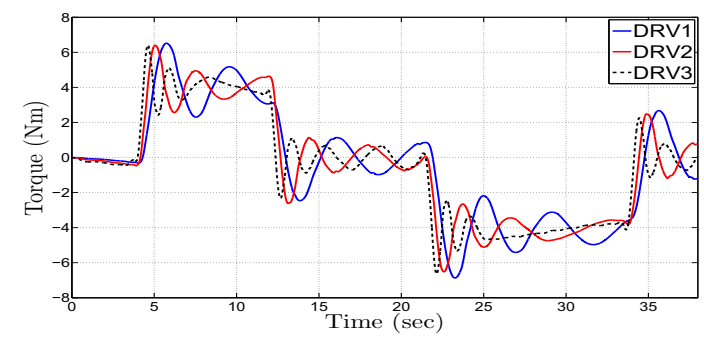

Fig. 7. Comparison of generated driver torques, $T_{\mathrm{dr}}$, for the three drivers.

\section{B. MPC Design}

The MPC design requires a prediction model. Since MPC is a discrete-time control methodology, we convert the abovementioned model to discrete-time with sampling period $t_{s}=$ 50 ms. Hence, we have,

$$
\begin{aligned}
x(k+1) & =A^{d} x(k)+B^{d} u(k)+E^{d} w(k), \\
y(k) & =C^{d} x(k),
\end{aligned}
$$

where again $x=\left(\omega_{s}, \delta_{s}, \beta, r, \psi, \Delta \psi, \Delta y\right)^{\top}$ for the case of the system without the driver, and $x=$ $\left(\omega_{s}, \delta_{s}, \beta, r, \psi, \Delta \psi, \Delta y, x_{d 1}, x_{d 2}, x_{d 3}\right)^{\top}$ for the case of the system with the driver included. In addition, $u=T_{\text {con }}$, and $w=\rho_{\text {ref }}$, and $y=\Delta y$.

In contrast to the lateral preview control for lateral control of vehicles where the path curvature is assumed to be known a priori, here the path curvature is constant over the prediction horizon, i.e., for $h \in \mathbb{Z}_{+}$,

$$
\rho_{\text {ref }}(h \mid k)=\rho_{\text {ref }}(k),
$$

where $\rho_{\text {ref }}(k)$ is the curvature measured at this particular time instant. Next, we augment the system dynamics with the reference path curvature $\rho_{\text {ref }}$. Hence, the MPC prediction model is given by

$$
x_{p}(k+1)=A^{p} x_{p}(k)+B^{p} u_{p}(k),
$$

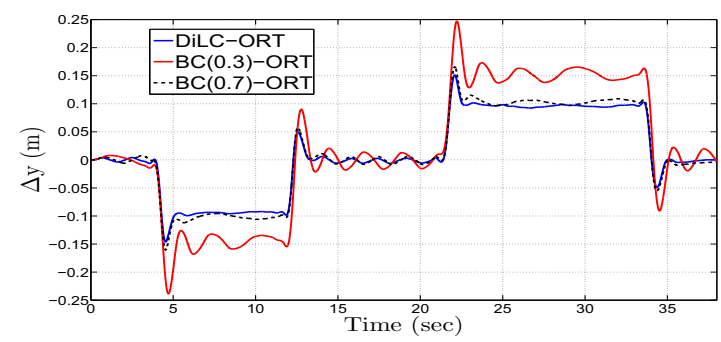

Fig. 8. Comparison of $\Delta y$ for the ORT-assisted cases of DRV2.
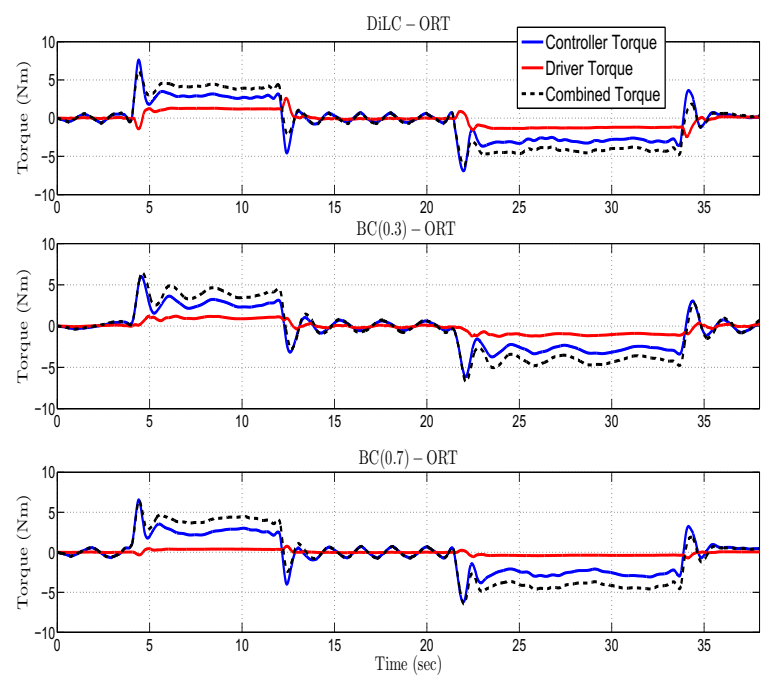

Fig. 9. Time histories of $T_{\mathrm{con}}, T_{\mathrm{dr}}$, and $T_{\mathrm{tot}}$ for the ORT assisted cases of DRV2.

where

$$
A^{p}=\left[\begin{array}{cc}
A^{d} & E^{d} \\
0 & 1
\end{array}\right], B^{p}=\left[\begin{array}{c}
B^{d} \\
0
\end{array}\right] .
$$

The main idea of the MPC control design is that a finite horizon optimal control problem is solved at every control cycle. Here we enforce only input constraints, hence the MPC scheme is as follows

$$
\begin{array}{cl}
\min _{U_{N}(k)} & \sum_{h=0}^{N-1} x_{p}(h \mid k)^{\top} Q x_{p}(h \mid k)+u_{p}(h \mid k)^{\top} R u_{p}(h \mid k)(3 \\
\text { s.t. } & x_{p}(h+1 \mid k)=A^{p} x_{p}(h \mid k)+B^{p} u_{p}(h \mid k) \\
& u_{\min } \leq u_{p}(h \mid k) \leq u_{\max }, h=0, \ldots, N_{u}-1 \\
& u_{p}(h \mid k)=0, \quad h=N_{u}, \ldots, N-1
\end{array}
$$

where $U_{N}(k)=\left(u_{p}(0 \mid k), \ldots, u_{p}(N-1 \mid k)\right)$. It is possible for the prediction horizon $N$ to differ from the control horizon $N_{u}$, the number of free control moves to be chosen.

In order to enforce tracking of the reference path, the lateral offset $\Delta y$ needs to be minimized, thus the cost function in (31a) is

$$
J=\sum_{h=0}^{N-1} q_{r} \Delta y^{2}(h \mid k)+q_{u} u^{2}(h \mid k) .
$$

The values of the MPC parameters are the same for both the $\mathrm{BC}(\lambda)$ and the DiLC controllers; $q_{r}=200, q_{u}=0.1$, $N=21, N_{u}=12$, and $u_{\max }=-u_{\min }=8 \mathrm{Nm}$.

\section{Simulations}

Our main objective is to evaluate the performance of the controllers developed in Section IV, and compare the driver effort during an unassisted and an assisted lane-tracking maneuver. Three different sets of data for the driver model were used (see Table I) in order to test different driver styles. All controller designs were based on the data corresponding to DRV2. Note that during testing the full driver model (that is, including the kinesthetic part and transport delay) was used in all cases. Also, all numerical simulations were performed with CarSim ${ }^{\circledR}$ [14] using the numerical values of the test vehicle and the steering column parameters shown 
in Table II. The longitudinal speed of the vehicle is assumed to be $V_{x}=15 \mathrm{~m} / \mathrm{s}(54 \mathrm{~km} / \mathrm{h})$, and the curvature of the path is given by a sensor on the vehicle which is provided directly by $\operatorname{CarSim}^{\circledR}$. Since this signal is noisy, pre-filtering is applied prior to its utilization by the controller.

\section{A. Driver-only Case}

Simulations of unassisted drivers were performed using the three driver data sets shown in Table I. In Figure 6, we observe that DRV3 manages to perform better tracking compared to the other two drivers, and with a less oscillatory response of $\Delta y$. On the other hand, DRV1 has a very sluggish response, whereas the reaction of DRV2 is somewhere in between that of DRV1 and DRV3.

Figure 7 presents the time histories of the driver torques for the same maneuver. All drivers provide approximately equal magnitudes of torque but there is a difference among the torque histories in terms of phasing, with DRV1 and DRV2 being more oscillatory compared to the torque history of DRV3.

TABLE I: Parameter values for the driver models.

\begin{tabular}{|c|c|c|c|}
\hline Values & DRV1 & DRV2 & DRV3 \\
\hline$T_{N}[\mathrm{sec}]$ & 0.12 & 0.12 & 0.12 \\
\hline$t_{p}[\mathrm{sec}]$ & 0.1 & 0.06 & 0.04 \\
\hline$K_{a}$ & 22 & 30 & 45 \\
\hline$K_{c}$ & 14 & 20 & 27 \\
\hline$T_{L}[\mathrm{sec}]$ & 1.6 & 2.4 & 3.5 \\
\hline$T_{I}[\mathrm{sec}]$ & 0.35 & 0.2 & 0.1 \\
\hline$K_{D}$ & 1 & 1 & 1 \\
\hline$T_{1}[\mathrm{sec}]$ & 2.5 & 4.5 & 5.1 \\
\hline$K_{G}$ & -0.63 & -0.85 & -0.63 \\
\hline$T_{k_{1}}[\mathrm{sec}]$ & 1.99 & 2.99 & 3.99 \\
\hline$T_{k_{2}}[\mathrm{sec}]$ & 0.013 & 0.043 & 0.013 \\
\hline
\end{tabular}

Next, we evaluate the performance of the proposed control designs for two different scenarios. In the first scenario, the numerical values of the driver parameters of the DiLC controllers match those of the parameters of the actual driver,

TABLE II: Numerical values of the vehicle and steering column parameters.

\begin{tabular}{|c|c|}
\hline Vehicle Parameter & Parameter Numerical Value \\
\hline$m[\mathrm{~kg}]$ & 1653 \\
\hline$\ell_{F}[\mathrm{~m}]$ & 1.402 \\
\hline$\ell_{R}[\mathrm{~m}]$ & 1.646 \\
\hline$I_{z}\left[\mathrm{kgm}^{2}\right]$ & 2765 \\
\hline$C_{f}[\mathrm{~N} / \mathrm{rad}]$ & 42000 \\
\hline$C_{r}[\mathrm{~N} / \mathrm{rad}]$ & 81000 \\
\hline$\eta_{t}[\mathrm{~m}]$ & 0.225 \\
\hline$J_{s}\left[\mathrm{kgm}^{2}\right]$ & 0.11 \\
\hline$g_{s}$ & 16 \\
\hline$b_{s}[\mathrm{Nm} / \mathrm{rad} / \mathrm{sec}]$ & 0.57 \\
\hline$K_{p}$ & 0.038 \\
\hline \multicolumn{2}{|c}{} \\
\hline
\end{tabular}

namely those of DRV2, and the controllers' performance is evaluated against the driver-only case and against the $\mathrm{BC}(\lambda)$ controllers. In the second scenario, the DiLC controllers are tested using an incorrect driver model (namely, DRV1 and DRV3).

\section{B. Driver-Assist Case: Correct Driver Model}

In this section we present the results when driver DRV2 is being assisted by the ORT-based lane-tracking controller (Section IV-A). Figure 8 shows the error $\Delta y$ for the DiLCORT, BC(0.3)-ORT and BC(0.7)-ORT cases, respectively. The performance improvement in terms of regulating $\Delta y$ is significant for the DiLC-ORT and the $\mathrm{BC}(0.7)$-ORT cases, compared to the $\mathrm{BC}(0.3)$-ORT, and, especially, to the driveronly case (compare with Figure 6).

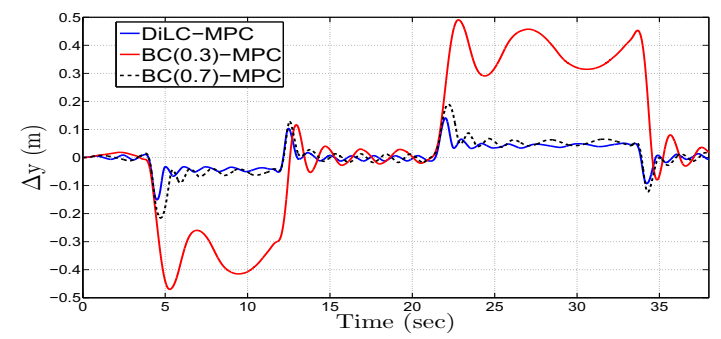

Fig. 10. Comparison of $\Delta y$ for the MPC assisted cases of DRV2.

The torques $T_{\text {con }}, T_{\mathrm{dr}}$, and $T_{\text {tot }}$ for all ORT-assisted cases are shown in Figure 9. One sees that for all the ORT-assisted cases the amount of torque generated by the driver is greatly decreased compared to the non-assisted case (compare with Figure 7).
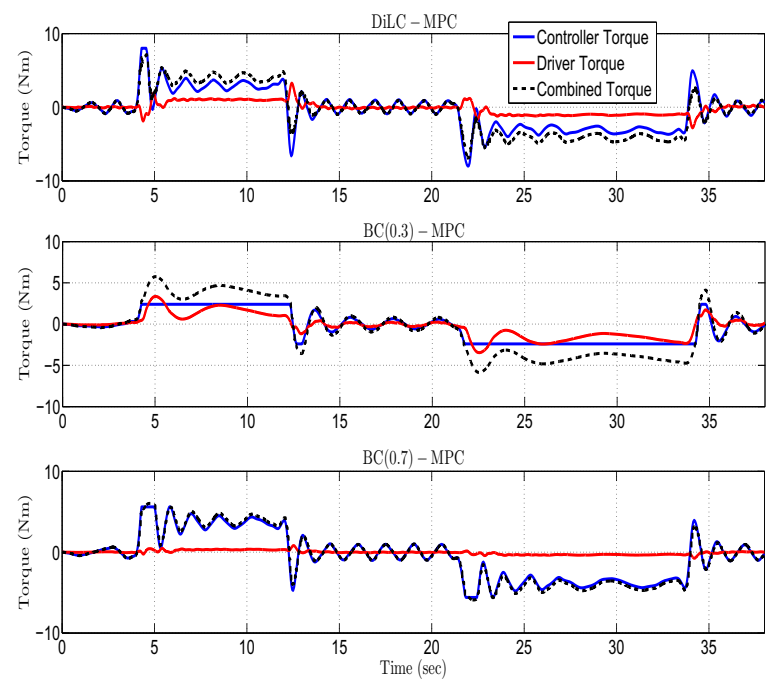

Fig. 11. Time histories of $T_{\mathrm{con}}, T_{\mathrm{dr}}$, and $T_{\mathrm{tot}}$ for the MPC assisted cases of DRV2.

Next, we present the results for the MPC-assisted driver case. The response of $\Delta y$ for DRV2 are presented in Figure 10. Similarly to the ORT approach, the tracking performance is significantly improved for the DiLC-MPC and $\mathrm{BC}(0.7)-\mathrm{MPC}$ designs, compared to other cases and, 
in particular, the driver-only case. It is interesting to note that with the use of this control design the $\mathrm{BC}(0.3)$-MPC controller does not enhance tracking performance to the same extent as the $\mathrm{BC}(0.3)-\mathrm{ORT}$. Compared to the other designs, the tracking performance of DiLC-MPC is the best one in terms of maximum steady-state values of $\Delta y$.

The generated torques for the MPC-based steering-assist system for DRV2 are also shown in Figure 11. The driver torque for the DiLC-MPC and BC(0.7)-MPC cases is again very small compared to the driver-only case, whereas for the $\mathrm{BC}(0.3)-\mathrm{MPC}$ case the generated driver torque is higher and is closer to the driver-only case.

\section{Driver-Assist Case: Incorrect Driver Model}

In this scenario we investigate the robustness of the DiLC controllers with respect to the driver model parameter mismatch. Both MPC-based and ORT-based DiLC controllers (which were designed based on the parameters of DRV2) were tested using either the DRV3 parameters. Figure 12 shows the response of $\Delta y$ for the case of driver DRV3. The assisted-steering performance is improved for the case of DiLC-ORT, which has a smooth and satisfactorily performance. On the other hand, DiLC-MPC decreases $\Delta y$, but the oscillatory response is noticeable. Overall, we see that the MPC design performs very well when the driver model parameters are accurately known, but it is rather sensitive to any change in these parameters, at least compared to the ORT controller. These results imply that for implementing MPC-based designs it will be probably imperative to have accurate identification algorithms of the driver and perhaps even adapt the MPC design "on-the-fly" to these parameters.

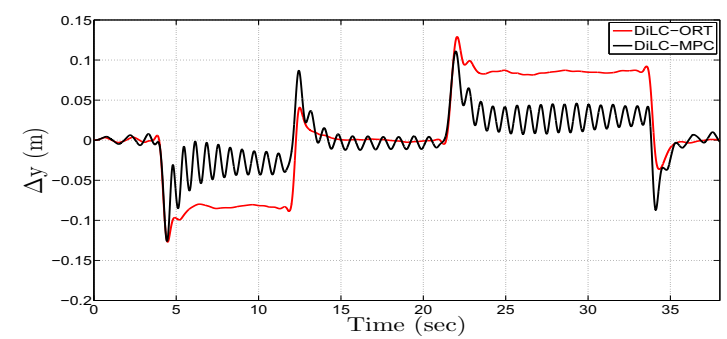

Fig. 12. Comparison of the $\Delta y$ responses for DiLC-ORT, and DiLC-MPC for DRV3.

\section{CONCLUSIONS}

In this paper we have investigated the benefits of designing a lane-tracking system with a suitable model of the driver in the loop. By incorporating a driver model, the controller can better predict and compensate for incorrect driver reaction. Two different design methodologies have been used and compared. One based on output feedback regulation theory, and the other based on model predictive control. It was shown that the benefits of including the driver model in the loop can be significant, even more when using an MPC design approach, although further investigation is required, in order to analyze the robustness of such a control design. The main challenge still remains on how to get good driver model parameters that adequately reflect the current driver capabilities, and how to design/adapt the controller "in-situ" in order to take advantage of a known driver model. In regards to the driver model parameter estimation or driver classification, some recent efforts have been reported in [17], [21].

Acknowledgment: This work has been supported by NSF award CMMI-1234286.

\section{REFERENCES}

[1] B. B. Litkouhi, A. Y. Lee, and D. B. Craig, "Estimator and controller design for lanetrak, a vision-based automatic vehicle steering system," in Proceedings of the 32nd IEEE Conference on Decision and Control, pp. 1868-1873 vol.2, December 1993.

[2] U. Özgüner, K. Ünyelioğlu, and C. Hatipoğlu, "An analytical study of vehicle steering control," in Proceedings of the 4th IEEE Conference on Control Applications, pp. 125-130, September 1995.

[3] J. Guldner, H.-S. Tan, and S. Patwardhan, "Study of design directions for lateral vehicle control," in Proceedings of the 36th IEEE Conference on Decision and Control, vol. 5, pp. 4732-4737, December 1997.

[4] C. C. MacAdam, "Application of an optimal preview control for simulation of closed-loop automobile driving," IEEE Transactions on Systems, Man and Cybernetics, vol. 11, no. 6, pp. 393-399, 1981.

[5] R. S. Sharp and V. Valtetsiostis, "Optimal preview car steering control," Supplements to Vehicle System Dynamics, vol. 35, pp. 101$117,2001$.

[6] R. Hess and A. Modjtahedzadeh, "A control theoretic model of driver steering behavior," Control Systems Magazine, vol. 10, pp. 3-8, August 1990.

[7] E. Donges, "A two-level model of driver steering control," Human Factors, vol. 20, no. 6, pp. 691-707, 1978.

[8] M. Land and D. Lee, "Where we look when we steer," Nature, vol. 369 , no. 6483, pp. 742-744, 1994.

[9] M. Land, Vision and Action, ch. The visual control of steering, pp. 163-180. Cambridge, UK: Cambridge University Press, 1998.

[10] D. D. Salvucci and R. Gray, "A two-point visual control model of steering.," Perception, vol. 33, no. 10, pp. 1233-48, 2004.

[11] C. Sentouh, P. Chevrel, F. Mars, and F. Claveau, "A sensorimotor driver model for steering control," in IEEE International Conference on Systems, Man and Cybernetics, pp. 2462 -2467, 2009.

[12] P. Gáspár, B. Németh, and J. Bokor, "Design of integrated vehicle control using driver models," in Robust Control Design, vol. 7, pp. 517-522, 2012

[13] L. Saleh, P. Chevrel, F. Claveau, J.-F. Lafay, and F. Mars, "Shared steering control between a driver and an automation: Stability in the presence of driver behavior uncertainty," IEEE Transactions on Intelligent Transportation Systems, vol. 14, pp. 974-983, June 2013.

[14] CarSim, CarSim, version 8. Ann Arbor, Michigan: Mechanical Simulation Inc., 2009. (www.carsim.com).

[15] J. Ackermann, A. Bartlett, D. Kaesbauer, W. Sienel, and R. Steinhauser, Robust Control: Systems with Uncertain Physical Parameters. Springer, 1993.

[16] Y. J. Hsu and J. C. Gerdes, "A feel for the road : A method to estimate tire parameters using steering torque," in International Symposium on Advanced Vehicle Control, (Taiwan,Taipei), pp. 1-6, 2006.

[17] J. Steen, H. J. Damveld, R. Happee, M. M. van Paassen, and M. Mulder, "A review of visual driver models for system identification purposes," in IEEE International Conference on Systems, Man, and Cybernetics, pp. $2093-2100,2011$.

[18] Y. Yao, "Vehicle steer-by-wire system control," SAE Technical Paper 2006-01-1175, 2006.

[19] B. A. Francis, "The linear multivariable regulator problem," in IEEE Conference on Decision and Control, vol. 15, pp. 873 -878, December 1976.

[20] C. E. Garcia, D. M. Prett, and M. Morari, "Model predictive control: Theory and practicea survey," Automatica, vol. 25, no. 3, pp. 335-348, 1989.

[21] J. Lu, D. Filev, K. Prakah-Asante, F. Tseng, and I. Kolmanovsky, "From vehicle stability control to intelligent personal minder: Realtime vehicle handling limit warning and driver style characterization," in Computational Intelligence in Vehicles and Vehicular Systems, pp. $43-50$, April 2009. 